Title page

Inhibition of Viral Macrodomain of COVID-19 and Human TRPM2 by losartan

Azhar Sallari Jazzi ${ }^{1}$, Karim Mahnam ${ }^{2}$, Sayed Hossein Hejazi ${ }^{3}$, Mohammad Sadegh Damavandi ${ }^{1}$, Parisa Sadeghi ${ }^{1}$, Mehrdad Zeinalian ${ }^{4}$, Faezeh Tabesh ${ }^{5}$, Seyedeh Mahnaz Mirbod ${ }^{6}$, Hossein Khanahmad $^{4 *}$

1. Ph.D. student of medical bacteriology, Department of Microbiology, School of Medicine, Isfahan University of Medical Sciences, Isfahan, Iran.

2. Associate Professor of Biophysics, Biology Department, Faculty of Sciences, Shahrekord University, Shahrekord, I.R. Iran.

3. Professor of Parasitology, Skin Diseases and Leishmaniosis Research Center, Department of Parasitology and Mycology, School of Medicine, Isfahan University of Medical Sciences, Isfahan, Iran.

4. MD-Ph. D, Department of Genetics and Molecular biology, School of Medicine, Isfahan University of medical science, Isfahan, Iran.

5. Cardiac Rehabilitation Research Center, Cardiovascular Research Institute, Isfahan University of Medical Sciences, Isfahan, Iran.

6. Resident of Cardiology, Department of cardiology, Isfahan University of Medical Sciences, Isfahan, Iran.

Corresponding Author; Hossein Khanahmad

MD-Ph. D, Department of Genetics and Molecular biology, School of Medicine, Isfahan University of medical science, Isfahan, Iran

Tel: $\quad+983136680048$

Fax: $\quad+98316688597$

E-mail: damavand.mohammad@yahoo.com 


\title{
Inhibition of Viral Macrodomain of COVID-19 and Human TRPM2 by losartan
}

\begin{abstract}
Background: The SARS-CoV-2 virus is a new highly contagious Coronavirus with a positivesense RNA encoding 16 non-structural proteins (nsps16, nsp15, nsp3). In this study, the coronavirus pathogenicity and the losartan functional ligand for inhibiting TRPM2 and macrodomain have been molecularly evaluated.
\end{abstract}

Material and method: In this study, the structures of macrodomain binding ADP ribose in CoVs and human Transient Receptor Potential Cation Channel Subfamily M Member 2 (TRPM2) protein were downloaded from protein data bank. Then, a virtual screening was done to recognize the hit compounds from GalaXi_2019-10, KnowledgeSpace_2019-05, and REALspace_2019-12 databases. This collection, then, was imported to the ligandScout software, on the base of Adenosine Diphosphate Ribose (ADPR) pharmacophore model.

Result: Among seven compounds, five compounds were finally evaluated as the structural analogs of ADPR or other nucleotides, from which one compound was a non-FDA-approved sulphonamide and was removed. The other compound, losartan, was finally selected for molecular docking and molecular dynamic simulation. According to the virtual screening and docking, losartan was candidate as an effective ligand for TRPM2 and macrodomain.

Conclusion: In the current study losartan earned a proper dock score and binding affinity to create the complexes with TRPM2 and macrodomain. The inhibitory effect of losartan on PARP has been shown and it could interfere positively in several points (PARP, PARG- macrodomain and TRPM2) and decreases oxidative stress and apoptosis in COVID-19.

Key words: Viral Macrodomain; COVID-19; TRPM2; losartan; Bioinformatics 


\section{Introduction}

At the end of 2019, the emergence of the novel coronavirus infection in Wuhan, China, it has rapidly spread across China and many other countries. The International Committee on Taxonomy of Viruses has been named SARS-CoV-2 and the disease it causes has been named coronavirus disease 2019 (COVID-19). The SARS-CoV-2 virus is a new pathogen that is highly contagious, which can spread quickly (1). This virus must be considered capable of causing enormous health, economic and societal impacts with their setting The SARS-CoV-2 virus like SARS-CoV and MERS-CoV, is a beta-coronavirus which all of them have originated from bats. On 30 January 2020, the World Health Organization (WHO) declared that the SARS-CoV-2 outbreak constituted a Public Health Emergency of International Concern (2). The outbreak has rapidly evolved, and more than 180 countries worldwide have reported laboratory-confirmed cases of SARS-CoV-2 (3).

Coronaviruses (COVs) are positive-sense enveloped RNA viruses which are larger than the other RNA viruses, encoding 16 non-structural proteins (nsps). They have different proteins that are important for preventing and blocking the innate immune response, including enzymes such as an endoribonuclease (nsp15), an O-methyl transferase (nsp16), and an macrodomain with ADPribosyl hydrolase (nsp3) (4). The macrodomain of nsp3 harbors several conserved domains in which some enzymatic activities have been attributed. COVs macrodomain has a close functional association with poly (ADP-ribose) polymerases (PARPs), which is known as the regulator of apoptosis in eukaryotic cells. PARPs regulate important cellular processes such as cell-cycle progression, cell division, host-virus interaction, DNA damage repair, genome maintenance, transcription regulation, protein degradation, ageing, and cell death. PolyADP-ribosylation can be removed by the action of two divergent enzymes, poly (ADP-ribose) glycol hydrolase (PARG) and ADP-ribosyl hydrolase 3 (ARH3) $(5,6)$. PARG is a catabolic enzyme that cleaves 
ADP-ribose (ADPR) polymers that synthesized by members of the PARPs family of enzymes. The Viral macrodomains involve in cellular signaling consist of; ADP-ribose-generating orconsuming pathways and roles in determining RNA synthesis, replication, and virulence. This protein hydrolase ADPR polymer from ADP-ribosylated proteins. ADPR-binding to the CoVs nsp3 macrodomain that is necessary for the initiation of virus replication, whereas the hydrolase activity facilitates to amplify the replication complex. Therefore, macrodomains can remove ADP-ribosy polymer from modified proteins (7).

The antiviral properties of PARPs are regulated by interferon (IFN), and under evolutionary selection, it has been given that primary function of viral macrodomain is counter the host response viral infection. Accordingly, macrodomain by removing ADP-ribosylation from modified host proteins, prevent host responses to viral infection(8).

On the other hand, PARG activity of MD leads to increase the level of free ADPR in the cell, which activates the Transient Receptor Potential 2 (TRPM2). TRPM2, a subtype of TRPmelastatin subfamily (TRPM), is a multifunctional $\mathrm{Ca}^{2+}$ permeable and nonselective cation channel. Exposure to hydrogen peroxide $\left(\mathrm{H}_{2} \mathrm{O}_{2}\right)$, tumor necrosis factor $\alpha$ (TNF $\left.\alpha\right)$, reactive oxygen and nitrogen species is generally accepted to promote channel activation through the generation of ADPR, an agonist for TRPM2 (9). It performs as an important $\mathrm{Ca}^{2+}$ signaling regulator in a variety of cells, contributing to cellular functions including cytokine production, insulin release, cell motility, oxidative stress and cell death. TRPM2 activation cause to induce $\mathrm{Ca}^{2+}$ influx, and providing positive feedback for channel activation, led in necrosis and cell death (9). The Severe inflammatory response, cytokine storms, and necrosis in the patient's lungs with COVID-19, can explain the activation of TRPM2 signaling pathway, which can result the action of macrodomain or stress conditions that lead to accumulation of ADPR. Therefore, both macrodomain and TRPM2 inhibitors blocked calcium influx through TRPM2, inhibiting the 
cytokine storms, tissue necrosis, protecting cells from plasma membrane damage and cell death $(9,10)$.

Losartan is an angiotensin-converting enzyme (ACE) inhibitors that used to treat hypertension. ACE inhibitors are used for a similar indication but associated with a cough (11). Here, we showed that Losartan is a functional ligand for inhibition of the TRPM2 and macrodomain and has a high affinity to them. The comparative data have shown Losartan is effective agent for inhibition of the TRPM2 and macrodomain.

\section{Methods}

\section{Protein and ligand preparation}

High-resolution X-ray diffraction SARS CoV-2 of macrodomain binding ADP ribose phosphate and human TRPM2 protein structures were downloaded from protein data bank (https://www.rcsb.org/pdb). The exported files included 6W02 consists of two COVID-19 macrodomain molecules complexes with ADP ribose phosphate as the main ligand. Because macrodomain has only one monomer, we elected, chain A of the PDB structure, and other additional parts were omitted. Another exported file, 6PUR contains four TRPM2 molecules complexes with ADP ribose phosphate. Among the four molecules, chain A was selected, and extra parts were removed. In chain A, sequence between residue 1234 to 1534 was selected because it forms biding site of TRPM2, and this sequence which has been located in the cytoplasmic part involved in the cell cascade $(12,13)$.

All hydrogen atoms were added by the Discovery Studio software 2.5 (DS, Accerlys Inc, San Diego) and the proteins were prepared for the docking and virtual screening. Energy minimization was performed through a simulation module of Discovery Studio by conjugate gradient method and CHARMm force field $(12,13)$. This process was done with energy gradient 
of 0.1 cal $\AA^{-1}$. Ligands' 3D structures were prepared and minimized by the Marvin Suite software (14).

\section{Chemical library}

The procedure of this study shown the Figure 1. The virtual screening was done to recognition of the hit compounds from the GalaXi_2019-10, KnowledgeSpace_2019-05, and REALspace_2019-12 databases in biosolveit (https://www. biosolveit.de/) with infinisee software (15), for finding similar compounds to ADP ribose phosphate. The all current databases collections include up to $2.9 \times 10^{14}$ compounds, which consist of every compound on the world, but in this study on the base of the necessity and urgency of investigation about coronavirus treatment was more focused on the GalaXi_2019-10 database that has drug-like and all drug library (other databases is not removed).

Primary virtual screening was performed over all databases by the infiniSee software (ligand base, similarity 30-100), resulted in to obtain 20 compounds. This collection was imported to the ligandScout software and converted to ligandScout library, then, on the base of the ADPR pharmacophore model (two hydrogen donors and six hydrogen acceptors) (figure2) secondary virtual screening was done. After that, seven compounds were obtained by high pharmacophorefit score. Figure 2 Pharmacophore model of molecules that are similar to ADPR and pharmacophore of ADPR

Five of seven obtained compounds, were a structural analog of ADPR or other nucleotides, so these compounds were removed due to the potential toxic effects on human DNA and RNA polymerase. One compound was sulphonamide which was removed due to not being FDAapproved drug. The final compound, i.e. Losartan was finalized for the molecular docking step. 


\section{Molecular docking}

In this step, we used SeeSAR 9.2, AutoDock Vina module of ligandScout 4.3, and Molegro Virtual Docker v6.0 to investigate the conformational of Losartan in interaction with macrodomain and TRPM2 proteins $(16,17)$. At first SeeSAR 9.2 was used for a primary docking of Losartan. The applied parameters were used the genetic algorithm. The population size, selection pressure, and number of run were 100, 1.2 and 100, respectively. Conformations that were obtained in this step were used for final docking with Molegro Virtual Docker and AutoDock Vina, and the best affinity score was estimated between our load conformations (18, 19). The population size and the number of run in this step were 500 and 200 , respectively. Profiles of interactions between Losartan and proteins were obtained via Discovery Studio Visualizer and LIGPLOT software (20).

\section{Molecular dynamic simulation}

The molecular dynamic simulation was performed by AMBER18 package (21), and LEAP module of AMBER18 that was used for adding hydrogen to atoms. The ligand charges were generated by AM1-BCC method of Antechamber module. The topology of macromolecules was created by AMBER99SB-ILDN force field and tleap module of AMBER tools $(22,23)$. The generalized AMBER force field (GAFF2) was used for the ligand topology building with Antechamber module of AMBER18. Then the complexes were solvated in a truncated octahedral box of TIP3P. The water molecules with a buffer size of $10 \AA$, were neutralized by $\mathrm{Na}^{+}$or $\mathrm{Cl}^{-}$ ions for each complexes. The default protonation of Amber18 was used for titratable residues in protein. The energy minimization and MD simulations were used for each system with the sander module. Ten thousands cycles of minimization were performed with the steepest descent; and conjugate gradients for all parts of the systems were done to remove the bad steric interactions and get better minimum energy. Then, the position restraints at the constant volume 
(NVT) for $100 \mathrm{ps}$, with a restraint force of $10 \mathrm{kcal} / \mathrm{mol}$ at temperature of $100 \mathrm{~K}$, and then at constant pressure (NPT) for $100 \mathrm{ps,} \mathrm{with} \mathrm{a} \mathrm{restraint} \mathrm{force} \mathrm{of} 1 \mathrm{kcal} / \mathrm{mol}$ at temperature of $300 \mathrm{~K}$, were performed. Afterward, an equilibration step at NPT was performed for reaching to equilibrium in density for $100 \mathrm{ps,} \mathrm{while} \mathrm{the} \mathrm{restraint} \mathrm{force} \mathrm{was} \mathrm{removed.} \mathrm{This} \mathrm{method} \mathrm{allows} \mathrm{the}$ water to equilibrate around the complexes. In the position restraints and equilibration steps, Langevin dynamics were used to control the temperature. In final step, $20 \mathrm{~ns}$ (nanosecond) MD simulation was used for each protein-ligand complex. For analyses of MD simulations , CPPTRAJ of AMBER18 was used (24).

\section{Binding energy calculation}

An attractive and interesting method in Computer-Aided Drug Design (CADD) is Molecular Mechanics Poisson Boltzmann/ Surface Area (MM-PBSA). The binding energies ( $\Delta \mathrm{G}_{\text {binding }}=\mathrm{G}_{\text {complex }}-\mathrm{G}_{\text {protein }}-\mathrm{G}_{\text {ligand }}$ ) in this study were calculated with the MM-PBSA method by Amber18 (25). The accuracy of docking was calculated through obtaining the binding free energy over the molecular dynamics simulations. The last five ns of MD simulation trajectories were used for calculation of binding free energy (24).

After calculations of Gibbs energy changes $(\Delta \mathrm{G})$ and entropy changes $(\Delta \mathrm{S})$ values by the amber18 package, the enthalpy change $(\Delta \mathrm{H})$ was calculated $(\Delta \mathrm{G}=\Delta \mathrm{H}-\mathrm{T} \Delta \mathrm{S})$.

If the enthalpy change $(\Delta \mathrm{H})$ was negative numbers, the reactions between ligands and proteins would be exothermic.

\section{Results}

\section{The virtual screening for macrodomain and TRPM2}

After the primary virtual screening on the database by the infiniSee software, a collection containing of 20 ligands was obtained table1. Then, seven compounds were screened after 
pharmacophore modeling by ligandScout with pharmacophore-fit score 86.16 - 85.56 (table2 and fig2). Losartan (with pharmacophore-fit score 85.26) was between of them, and other compounds because were nucleotides or similar to nucleotides (such as guanosine ribose phosphate and other nucleotides or their analog) were removed, Losartan was selected as a hit compound.

\section{The docking results}

\section{ADP ribose and Losartan in the macrodomain}

The docking score ADP ribose phosphate and losartan in the Molegro Virtual Docker software for for macrodomain was calculated -207 and -197 , respectively (table3). The similar scores were also obtained using SeeSAR and Autodock Vina software tools. Losartan formed six hydrogen bonds with four amino acids, Phe132, Ser128, Gly180, and Ile131 by the distance between 1.83 and $2.44 \AA$ A. Besides, 20 hydrophobic contacts were exhibited with Asp22, Ile23, Phe156, Ala154, Val155, Lue164, Pro136, Pro125, Gly48, Gly51, Gly47, Gly46, Asn40, Ala39, His45, Leu127, Gly133 and Ile131 among the range 3.40-3.90 A. The highest hydrophobic interaction belonged to Ile131 with three hydrophobic interactions (figure 3 and table4).

\section{ADP ribose and Losartan in the TRPM2}

It was shown that ADP ribose phosphate, and losartan docking scores in the Molegro software were $\quad-149$ and -157 , respectively (table3). These ligands had a similar arrangement of score in both proteins in the Seesar and Autodock vina software tools. Losartan was exhibited four hydrogen binds with Asn1487, Arg1433 and Ser1382 residues within the range of $1.8-2.8 \AA$. A total of 13 hydrophobic bonds were found with Pro1388, Val1377, Leu1379, Ser1382, Ala1386, His1348, and Tyr1349 in the range of 3.1-3.9 $\AA$. The highest hydrophobic interactions were observed in Ala1386 with three contacts (figure 4 and table 4). 


\section{Molecular dynamic simulation}

For checking the stability of the protein-ligand complexes, $20 \mathrm{~ns}$ molecular dynamic simulations were used to all complexes. Figure 5 exhibits the RMSD value for every complex of trpm2 and macrodomain structures on the simulation time. Assessment of average last five ns of RMSD plots showed that they are relatively stable backbone trajectories of TRPM2-Lsr (average 3.44^, fig5B) and macrodomain-Lsr (average 2.16, fig5A) as compared to TRPM2-ADPR (average 4.2Å, fig5B), and macrodomain- ADPR (average $1.58 \AA$, fig5A) complexes. In addition, it can be inferred that creating losartan complex with these proteins is led to the stability of protein and it is a useful ligand for inhibition of them.

Figure 6 shows RMSF profile of these complexes. The residues of macrodomain have lower fluctuation in complex with ADPR relative to Losartan, but there are some peaks in the plot that are amino acids in the active site and had similar stability and same fluctuation as compared to macrodomain-ADPR fig6A, 6B, and. So it has concluded Losartan can contest with ADPR for binding macrodomain and create resemblance stability

In the case of TRPM2 protein, the Losartan induces less fluctuation or more stability relative to the ADPR inhibitor. (Figure 6B). These results are compatible with RMSD results (figure 5). In other words, despite of the more stability of the ADPR than Losartan, Losartan can compete to ADPR for creating a complex with macrodomain protein, and in the case of TRPM2, Losartan could be a proper inhibitor for TRPM2. The free binding energy and thermodynamics parameters of all ligands in complexes have been mentioned in table 5, it has shown that ADPR has more binding affinity for macrodomain, and Losartan has more binding affinity for TRPM2 protein. The binding between proteins and ligands were exothermic. These results also are similar to RMSD and RMSF results.

\section{Discussion}

The worldwide spread of the coronavirus became a severe and significant threat to human health. World health organization have announced that the coronavirus becomes a pandemic disease(13). As yet, there is not any precise treatment for coronavirus. In some articles have suggested that some of the protease inhibitors, remdisivir, and chloroquine are effective on the COVID-19, but 
have not shown an efficient and potent therapeutic effect on this virus. This limitation and threat made us work on some drug databases that are FDA approve and some drug-like databases. In the primary virtual screening by infinisee, the new bank had created by some molecules with relative similarity to ADPR. Next, pharmacophore modeling was shown seven molecules that have similar pharmacophore. Between them, because five compounds were analog of nucleotide, they were omitted. Nucleotides analog have toxicity for human cells and bodies, so were removed.

Criteria for selection of the ligand was six terms consist of:

1- visibility

2- FDA approves existed drugs

3- Availability

4- cost-benefit effective compounds

5- the relative similarity to ADPR but not capability to binding to other nucleotide-binding enzymes (on the other hand compound have selective toxicity for other enzymes, and their effect had been proved)

Above criteria and capability creating complex with macrodomain and TRPM2 (that assessed by docking, and MD simulations), Losartan was chosen as an effective ligand for these proteins. Results that were obtained from docking and molecular dynamic simulations emphasized that Losartan is a functional ligand for inhibition of the TRPM2, macrodomain and has a high affinity to them. Thermodynamic parameters revealed that losartan complexes most likely created spontaneously. Losartan had a decent dock score and binding affinity for creating complexes with TRPM2 and macrodomain. Besides, it can contest with ADPR for binding to these proteins and could disable the performance of the proteins. So TRPM2 and macrodomain became inactive and other signaling pathways that depend on them may be deactivated. 
Xiao Luo and et al. worked on the TRPM2 inhibitors by two novel ligands that were synthesized as ADPR analogs (26). Xiao Luo's work was done without attention to the possible toxicity of these compounds for every enzyme (enzymes that have a high affinity for binding to ADPR, such as DNA-polymerase or RNA-polymerase). Xiao lue and et al. had focused only on the synthesis of these compounds. That study conceded ADMET investigation and laboratory assays for these ligands in the future studies for other scientists.

Our study against the Xiao Lue et al study, was done with Precise focused on the possible toxicity, and utilization of the FDA approved drugs and drug-like database. The bioinformatic calculation was performed to increase the accuracy of screening methods and obtaining the best drug to inhibit the both proteins.

John G. Starkus and et al. studied scalaradial (natural product compounds) as an inhibitor of the TRPM2 with the experimental assay, but they have not any computational approaches (27). Scalaradial is not an available compound and, more importantly, is not as an FDA approved drug. Then there is an urgent necessity for drug and inhibition of the TRPM2 and macrodomain for treatment of any person affected by COVID-19 (28).

Our knowledge Until now, there was not any study for the inhibition of the macrodomain and its function. This study is for TRPM2 and macrodomain inhibition by a bioinformatics approach with the utilization of the drug and drug-like database. Particularly as now, there is no study for inhibition any coronavirus's macrodomain (that has high affinity to bind ADPR). It seems the activity of MD protein of COVID19 runs a lethal cycle between PARP, MD and TRPM2 Zeinalian, M., et al explained this cycle and suggested that PARP, MD (NSP3 of virus) and TRPM2 are critical therapeutic targets in COVID19 (29).

In this study, it has been focused on the coronavirus pathogenicity and mechanisms that how the human cell can combat them. It was concluded that viral macrodomain has the essential roles for 
the initiation of the cell infection and the virus-cell cycle. Macrodomain with poly ADPR degradation and increase of the free ADPR's levels can provide activation of the TRPM2 and prevent of the produce interferon-gamma so that cell would be susceptible to COVID-19 infection; therefore, it has been suggested as the target protein for efficient therapeutic approaches against the coronavirus infection.

Some different studies have shown that ACE inhibitors such as Losartan could have a protective effect on some tissue injury due to the systemic inflammatory diseases. Losartan has antiaggregative platelet, anti-diabetic, and prevent end organ injuries and fibrosis. Also in the disease that affected with chronic kidney disorder, and blood pressure, prescription losartan led to decrease from kidney damage. In another study has shown that Losartan was able to prevent damage to the ischemic and oxidative stress and proliferation of tubules cells and penetration of the macrophage from parenchyma kidneys (30). On the other hand, losartan could also reduce indirectly oxidative stress through inhibition of TRPM2. It leads to prevent cytokine storm, apoptosis, and cell necrosis. Losartan can also directly bind to TRPM2, and creates an inhibitory complex with it (31).

ADP-ribosylation alters the structure and functions of the protein and could be involved in several processes including genetic stability, DNA damage repair, chromatin remodeling, cellular stress response, and virus infection. PARG is a hydrolytic enzyme that commonly cleave the $\alpha\left(1^{\prime \prime}-2^{\prime}\right) O$-glycosidic linkages in PolyADP-ribose chains. This enzyme is believed to have both exo- and endo-glyco hydrolase activities, acting at terminal and internal sites of PolyADPribose chains, respectively. Thus, in addition to free ADPR, PARG generates the short chains of PolyADP-ribose which as a powerful cell death signa. TRPM2 activation by short chains of PolyADP-ribose or free ADPR results in calcium influx which make the cells become susceptible to cell death; whereas, inhibition of TRPM2 function enhances the cell survival (32). 


\section{Conclusion}

It seems Losartan can inhibit the viral macrodomain and TRPM2 so that it can interrupts the cell cycle of the virus and apoptosis, necrosis, fibrillation, and cytokine storm consequence of the COVID-19 infection. Therefore, Losartan, with preventing the induction of overexpression of the inflammatory cytokine, may decrease the severity of the disease and time of hospitalization. Presumably, Losartan with inhibition of these proteins activate the production of the interferongamma and intracellular defense against the COVID-19 virus, so enhancement resistance of person to the coronavirus.

References

1. Wu Y, Ho W, Huang Y, Jin D-Y, Li S, Liu S-L, et al. SARS-CoV-2 is an appropriate name for the new coronavirus. The Lancet. 2020;395(10228):P949-950.

2. Lu R, Zhao X, Li J, Niu P, Yang B, Wu H, et al. Genomic characterisation and epidemiology of 2019 novel coronavirus: implications for virus origins and receptor binding. The Lancet. 2020;395(10224):565-74.

3. Raoult D, Zumla A, Locatelli F, Ippolito G, Kroemer G. Coronavirus infections: Epidemiological, clinical and immunological features and hypotheses. Cell Stress.

4. Chan JF-W, Kok K-H, Zhu Z, Chu H, To KK-W, Yuan S, et al. Genomic characterization of the 2019 novel human-pathogenic coronavirus isolated from a patient with atypical pneumonia after visiting Wuhan. Emerging Microbes \& Infections. 2020;9(1):221-36.

5. Fehr AR, Jankevicius G, Ahel I, Perlman S. Viral macrodomains: unique mediators of viral replication and pathogenesis. Trends in microbiology. 2018;26(7):598-610.

6. Feng X, Koh DW. Roles of poly (ADP-ribose) glycohydrolase in DNA damage and apoptosis. International review of cell and molecular biology. 304: Elsevier; 2013. p. 227-81.

7. Poltronieri P. Emerging Concepts on the Role of ADP-Ribosylation. Multidisciplinary Digital Publishing Institute; 2020.

8. Grunewald ME, Chen Y, Kuny C, Maejima T, Lease R, Ferraris D, et al. The coronavirus macrodomain is required to prevent PARP-mediated inhibition of virus replication and enhancement of IFN expression. PLoS pathogens. 2019;15(5). 
9. Sumoza-Toledo A, Penner R. TRPM2: a multifunctional ion channel for calcium signalling. The Journal of physiology. 2011;589(7):1515-25.

10. Zheng M, Gao, Y., Wang, G. et al. Functional exhaustion of antiviral lymphocytes in COVID-19 patients. Cell Mol Immunol 2020.

11. Powers BJ, Coeytaux RR, Dolor RJ, Hasselblad V, Patel UD, Yancy WS, et al. Updated report on comparative effectiveness of ACE inhibitors, ARBs, and direct renin inhibitors for patients with essential hypertension: much more data, little new information. Journal of general internal medicine. 2012;27(6):716-29.

12. Tronrud D. Conjugate-direction minimization: an improved method for the refinement of macromolecules. Acta Crystallographica Section A: Foundations of Crystallography. 1992;48(6):912-6.

13. Brooks BR, Brooks III CL, Mackerell Jr AD, Nilsson L, Petrella RJ, Roux B, et al. CHARMM: the biomolecular simulation program. Journal of computational chemistry. 2009;30(10):1545-614.

14. Marvin was used for drawing, displaying and characterizing chemical structures, substructures and reactions, Marvin 17.21.0, ChemAxon (https://www.chemaxon.com).

15. infinisee version 1.3; BioSolveIT GmbH, Sankt Augustin, Germany, 2019, www.biosolveit.de/SeeSAR.

16. Wolber G, Langer T. LigandScout: 3-D pharmacophores derived from protein-bound ligands and their use as virtual screening filters. Journal of chemical information and modeling. 2005;45(1):160-9.

17. SeeSAR version 9.2; BioSolveIT GmbH, Sankt Augustin, Germany, 2019, www.biosolveit.de/SeeSAR.

18. Trott O, Olson AJ. AutoDock Vina: improving the speed and accuracy of docking with a new scoring function, efficient optimization, and multithreading. Journal of computational chemistry. 2010;31(2):455-61.

19. Thomsen R, Christensen MH. MolDock: a new technique for high-accuracy molecular docking. Journal of medicinal chemistry. 2006;49(11):3315-21.

20. Dassault Systèmes BIOVIA, Discovery Studio Modeling Environment, Release 2017, San Diego: Dassault Systèmes, 2016.

21. Salomon-Ferrer R, Case DA, Walker RC. An overview of the Amber biomolecular simulation package. Wiley Interdisciplinary Reviews: Computational Molecular Science. 2013;3(2):198-210. 
22. Lindorff-Larsen K, Piana S, Palmo K, Maragakis P, Klepeis JL, Dror RO, et al. Improved side-chain torsion potentials for the Amber ff99SB protein force field. Proteins: Structure, Function, and Bioinformatics. 2010;78(8):1950-8.

23. Da Silva AWS, Vranken WF. ACPYPE-Antechamber python parser interface. BMC research notes. 2012;5(1):367.

24. Farrokhnia M, Mahnam K. Molecular dynamics and docking investigations of several zoanthamine-type marine alkaloids as matrix metaloproteinase-1 inhibitors. Iranian journal of pharmaceutical research: IJPR. 2017;16(1):173.

25. Kumari R, Lynn A. Application of MM/PBSA in the prediction of relative binding free energy: Re-scoring of docking hit-list. Journal of Natural Science, Biology and Medicine. 2011;2(3):92-.

26. Luo X, Li M, Zhan K, Yang W, Zhang L, Wang K, et al. Selective inhibition of TRPM 2 channel by two novel synthesized ADPR analogues. Chemical biology \& drug design. 2018;91(2):552-66.

27. Starkus JG, Poerzgen P, Layugan K, Kawabata KG, Goto J-I, Suzuki S, et al. Scalaradial is a potent inhibitor of transient receptor potential melastatin 2 (TRPM2) ion channels. Journal of natural products. 2017;80(10):2741-50.

28. Wang M, Cao R, Zhang L, Yang X, Liu J, Xu M, et al. Remdesivir and chloroquine effectively inhibit the recently emerged novel coronavirus (2019-nCoV) in vitro. Cell research. 2020;30(3):269-71.

29. Zeinalian, M., Salari-Jazi, A., Jannesari, A., \& Khanahmad, H. (n.d.). A potential protective role of Losartan against coronavirus induced lung damage. <i>Infection Control \& Hospital Epidemiology, </i> 1-6. doi:10.1017/ice.2020.80

30. Ivanov M, Mihailović-Stanojević N, Milanović JG, Jovović Đ, Marković-Lipkovski J, Ćirović S, et al. Losartan improved antioxidant defense, renal function and structure of postischemic hypertensive kidney. PloS one. 2014;9(5).

301. Ko SY, Wang SE, Lee HK, Jo S, Han J, Lee SH, et al. Transient receptor potential melastatin 2 governs stress-induced depressive-like behaviors. Proceedings of the National Academy of Sciences. 2019;116(5):1770-5.

32. Lawarée E, Jankevicius G, Cooper C, Ahel I, Uphoff S, Tang CM. DNA ADPRibosylation Stalls Replication and Is Reversed by RecF-Mediated Homologous Recombination and Nucleotide Excision Repair. Cell Reports. 2020;30(5):1373-84. e4. 
Table 1. The molecular structures of 20 screened compounds by infiniSee program for coronavirus macrodomain and TRPM2

\begin{tabular}{llll}
\hline Compound's Names & Compound's structures & Compound's Names & Compound's structures \\
UDP-alpha-D-xylose & &
\end{tabular}

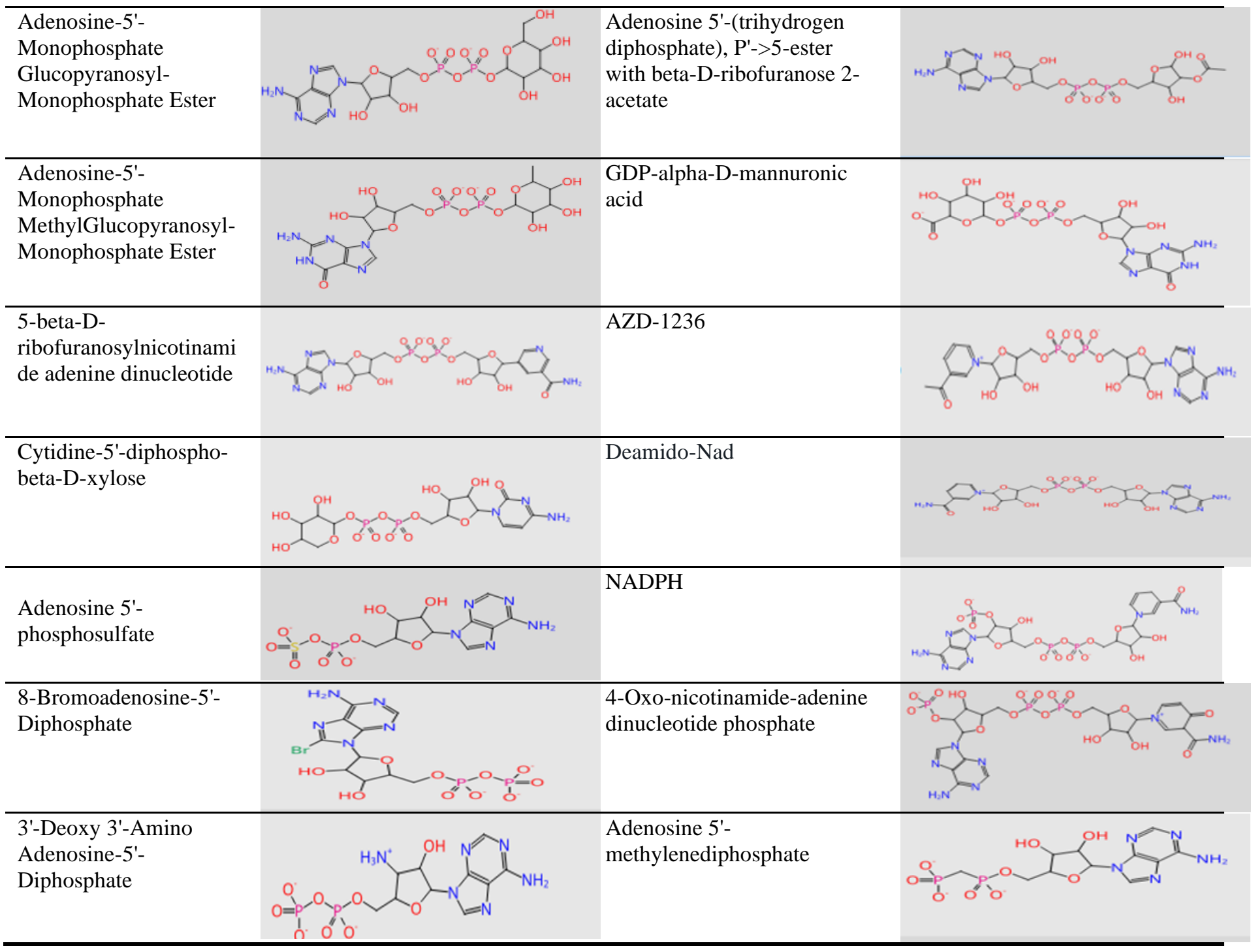




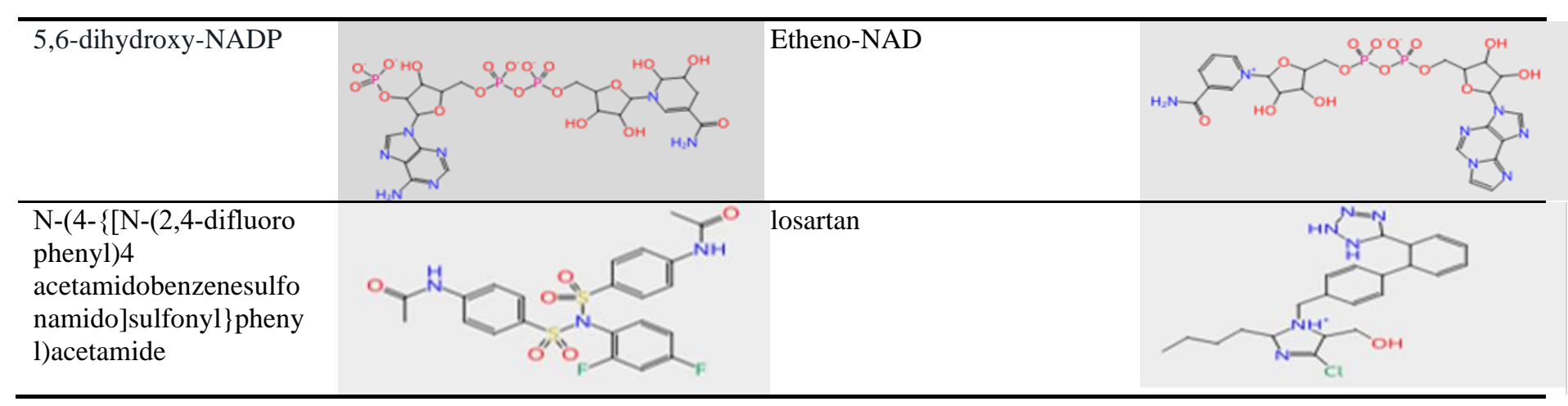

Table 2. six molecules that were removed after ligandScout virtual screening and Losartan selected for docking 


\begin{tabular}{|c|c|c|}
\hline Numbers & Compound's Names & Compound's Names \\
\hline 1 & $\begin{array}{l}\text { Adenosine-5'- } \\
\text { Monophosphate } \\
\text { Glucopyranosyl- } \\
\text { Monophosphate Ester }\end{array}$ & \\
\hline 2 & $\begin{array}{l}\text { 2'-Monophosphoadenosine- } \\
\text { 5'-diphosphoribose }\end{array}$ & \\
\hline 3 & $\begin{array}{l}\text { Adenosine-5'- } \\
\text { Monophosphate Methyl } \\
\text { Glucopyranosyl- } \\
\text { Monophosphate Ester }\end{array}$ & \\
\hline 4 & AZD-1236 & \\
\hline 5 & $\begin{array}{l}\text { GDP-alpha-D-mannuronic } \\
\text { acid }\end{array}$ & \\
\hline 6 & $\begin{array}{l}\mathrm{N}-(4-\{[\mathrm{N}-(2,4-\text { difluoropheny } \\
\text { 1)4- } \\
\text { acetamidobenzenesulfonami } \\
\text { do] sulfonyl }\} \text { phenyl }) \\
\text { acetamide }\end{array}$ & \\
\hline 7 & Losartan & \\
\hline
\end{tabular}

Table 3. The affinity and binding score of the ligand-protein complexes. 


\begin{tabular}{llll}
\hline Complex & Structures & MolDock Score & $\begin{array}{l}\text { AutoDock Vina } \\
\mathrm{kcal} / \mathrm{mol}\end{array}$
\end{tabular}

ADPR complex
with
macrodomain

losartan
complex with
macrodomain

macrodomain

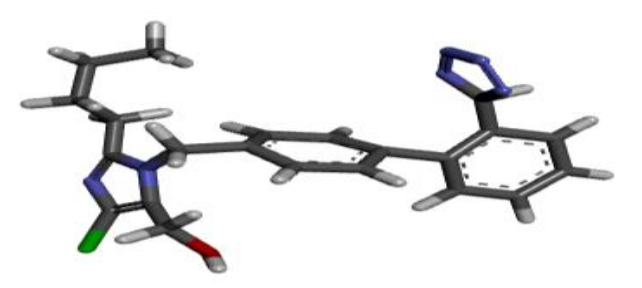

$-197$

$-20.30$

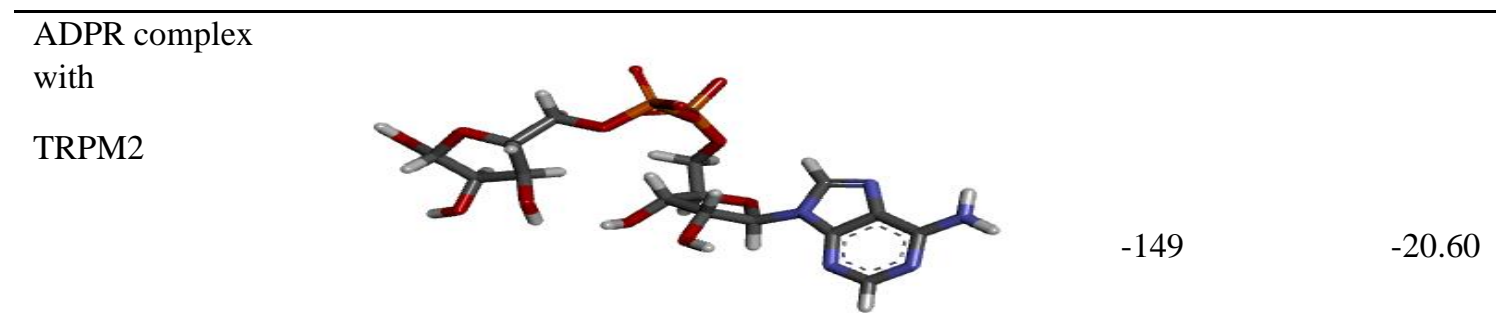

losartan

complex with

TRPM2

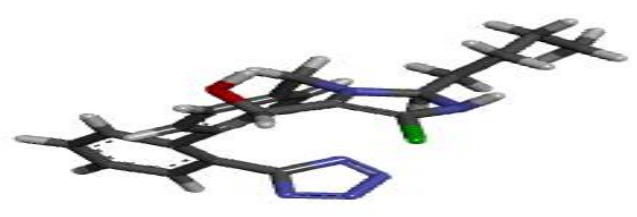

$-157$

$-17.30$

Docking score in Molegro Virtual Docker and docking affinity in the AutoDock Vina software tools.

Docking score and binding affinity of the Losartan is very close to ADPR, even higher than the ADPR. 
Table 4. Hydrogen bonding and hydrophobic interactions of the Complexes and distance of the

\begin{tabular}{|c|c|c|c|c|}
\hline \multirow[t]{2}{*}{ complex } & \multicolumn{2}{|c|}{ Hydrogen binds } & \multicolumn{2}{|l|}{ Hydrophobic interaction } \\
\hline & Amino acids & Distance $(\AA)$ & Amino acids & Distance $(\AA)$ \\
\hline $\begin{array}{l}\text { Macrodomain } \\
\text { complex with } \\
\text { Losartan }\end{array}$ & $\begin{array}{l}\text { Phe132, } \\
\text { Ser128, } \\
\text { Gly } 180, \\
\text { Ile131 }\end{array}$ & $1.83-2.44$ & $\begin{array}{l}\text { Asp22, Ile23, Phe156, Ala154, Val155, } \\
\text { Lue164, Pro136, Pro125, Gly48, Gly51, } \\
\text { Gly47, Gly46, asn40, Ala39, His45, } \\
\text { leu127, Gly133 }\end{array}$ & $3.40-3.90$ \\
\hline $\begin{array}{l}\text { TRPM2 complex } \\
\text { with Losartan }\end{array}$ & $\begin{array}{l}\text { Asn1487, } \\
\text { Arg1433, } \\
\text { Ser1382 }\end{array}$ & $1.8-2.8$ & $\begin{array}{l}\text { Pro1388, Val1377, Leu1379, Ser1382, } \\
\text { Ala1386, His1348,Tyr1349 }\end{array}$ & $3.1-3.9$ \\
\hline
\end{tabular}

interacting atoms between ligand and protein.

Table 5. Thermodynamic parameters of binding ligands to Macrodomain and TRPM2.

\begin{tabular}{lclc}
\hline complex & $\boldsymbol{\Delta} \mathbf{G M M}_{\mathbf{M B S A}}(\mathbf{k c a l} / \mathbf{m o l})$ & $\boldsymbol{\Delta S}(\mathbf{k c a l} / \mathbf{m o l})$ & $\mathbf{\Delta H}(\mathbf{k c a l} / \mathbf{m o l})$ \\
\hline Macrodomain-ADPR & -90.87 & -13.33 & -4063.21 \\
\hline $\begin{array}{l}\text { Macrodomain- } \\
\text { Losartan }\end{array}$ & -26.51 & -13.09 & -3927.33 \\
\hline TRPM2-ADPR & -16.13 & -13.10 & -3919.93 \\
\hline TRPM2- Losartan & -39.99 & -13.34 & -4015.31 \\
\hline
\end{tabular}




\section{STEP 1: primary virtual screening}

GALAXI- REALspace-KnowledgeSpace database

Infinisee program

$3 \times 10^{14}$

20

Step2:secondary virtual screening

20 convert to ldb drug bank for

ligandscout

Pharmacophore modeling

Pharmacophore-fit score

7

Select on the base

of the FDA

approved

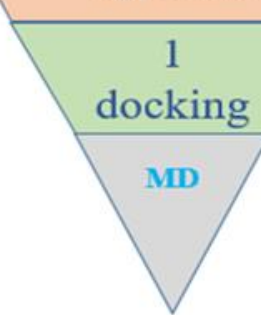

Figure 1. The procedure of this study. MD; Molecular dynamic simulation 


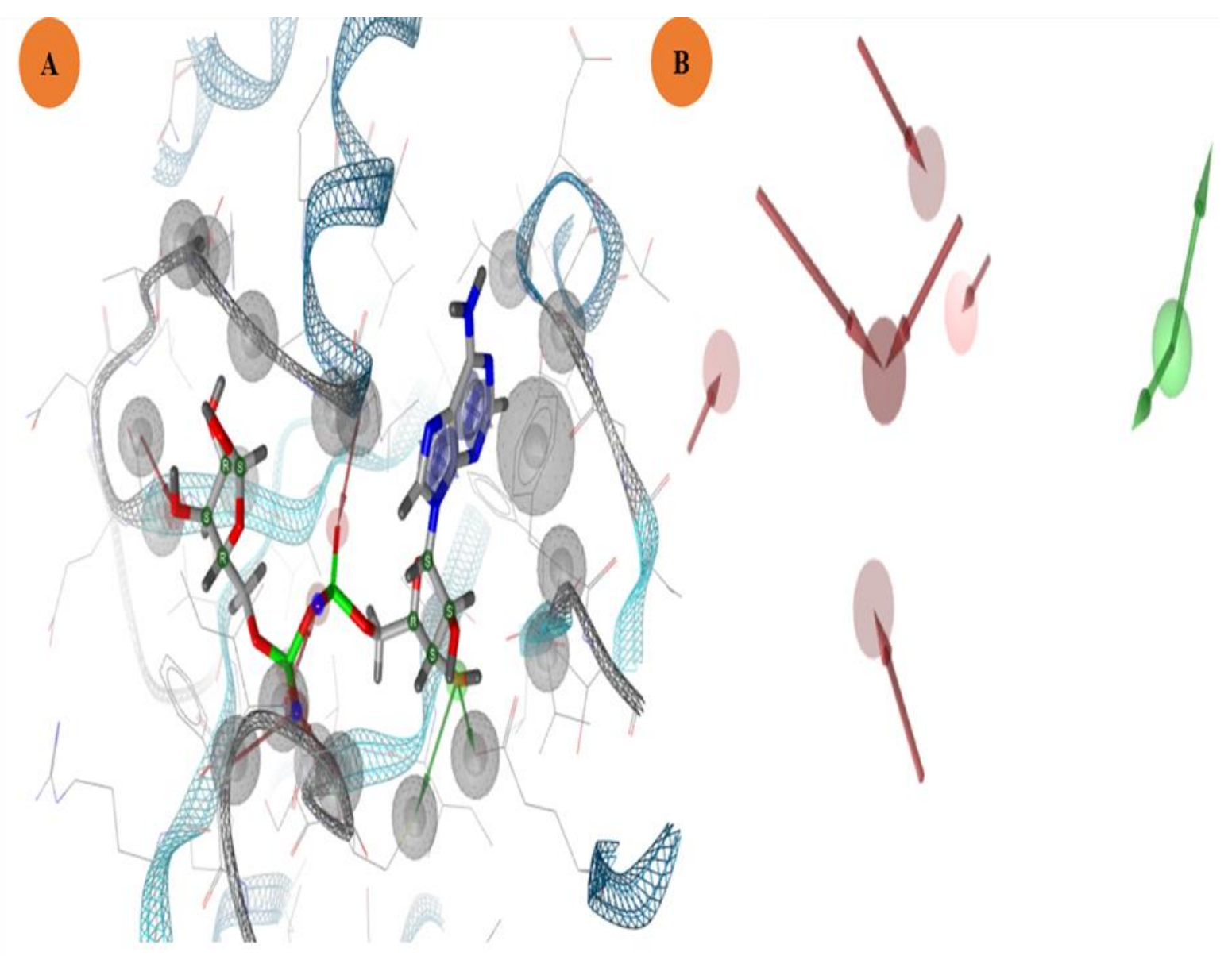

Figure 2. Pharmacophore model of ADPR. A-pharmacophore of the ADPR and its interaction with active site's amino acids macrodomain. B- pharmacophore model of ADPR which consist of two Hydrogen bond donor and six hydrogen bond acceptor 


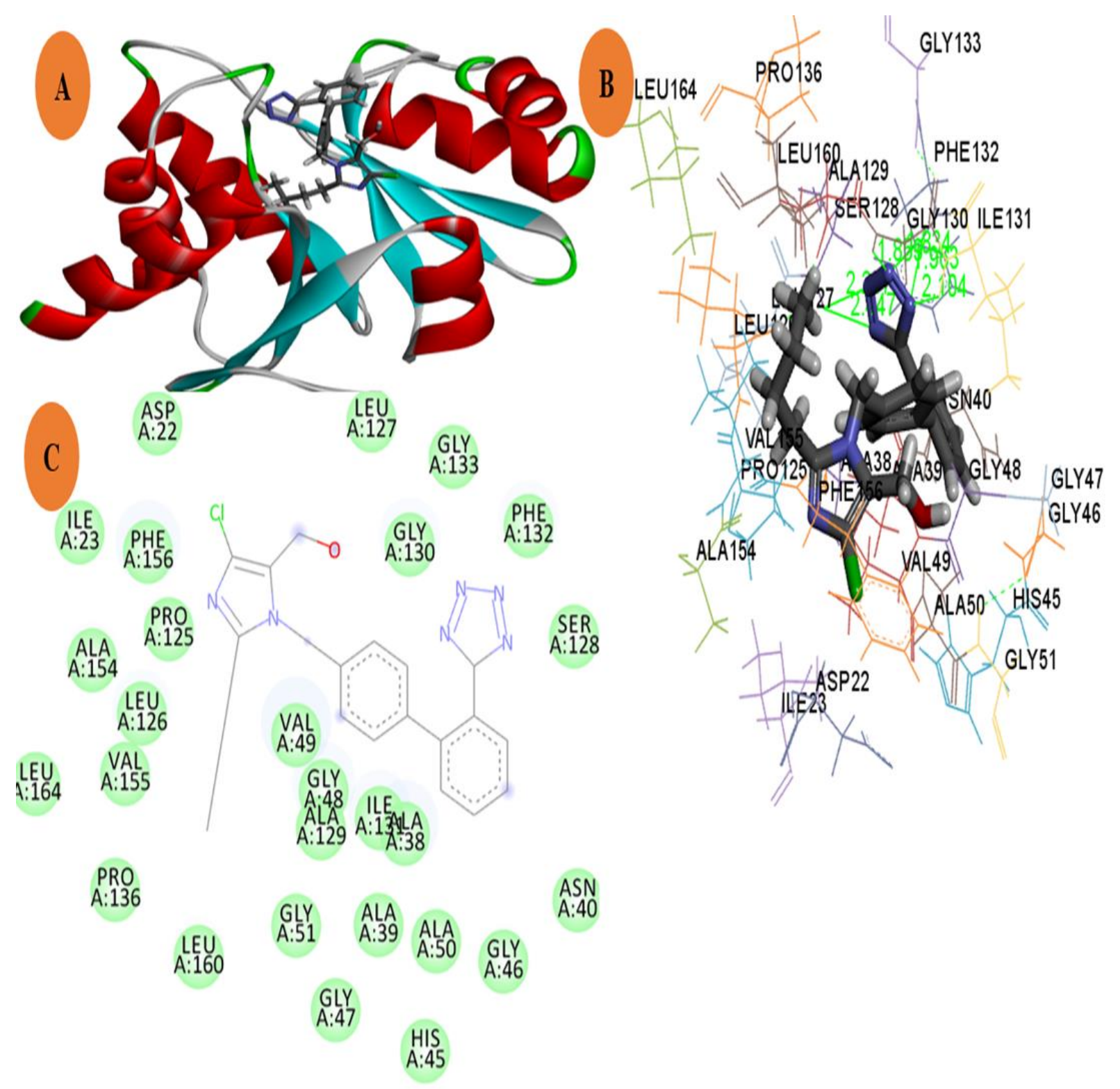

Interactions

$\square$ van der Woals

Figure 3. The predicted complex Macrodomain- Losartan binding mode. (A) The position of the Losartan in the active site of the macrodomain. (B) Hydrogen bond of Losartan with macrodomain in the active site (green line). (C) Van der Waals interaction between amino acids of active site and Losartan. 


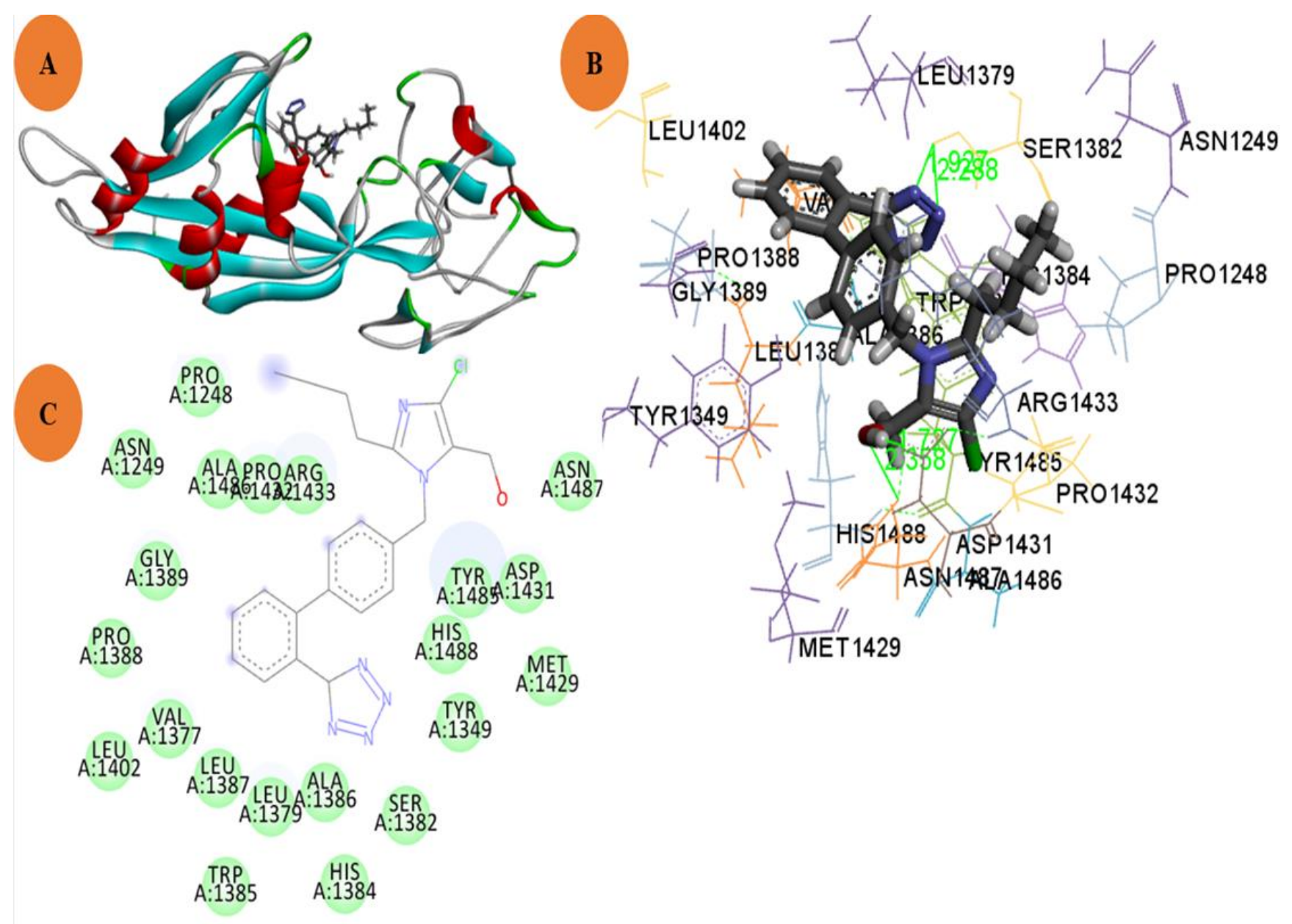

Interactions

$\square$ van der Waals

Figure 4. The predicted complex TRPM2-Lsr binding mode. (A) The position of the Losartan in the TRPM2'S active site. (B) Hydrogen bond of Losartan with TRPM2 in the active site (green line). (C) Van der Waals interaction between amino acids of the active site and Losartan. 

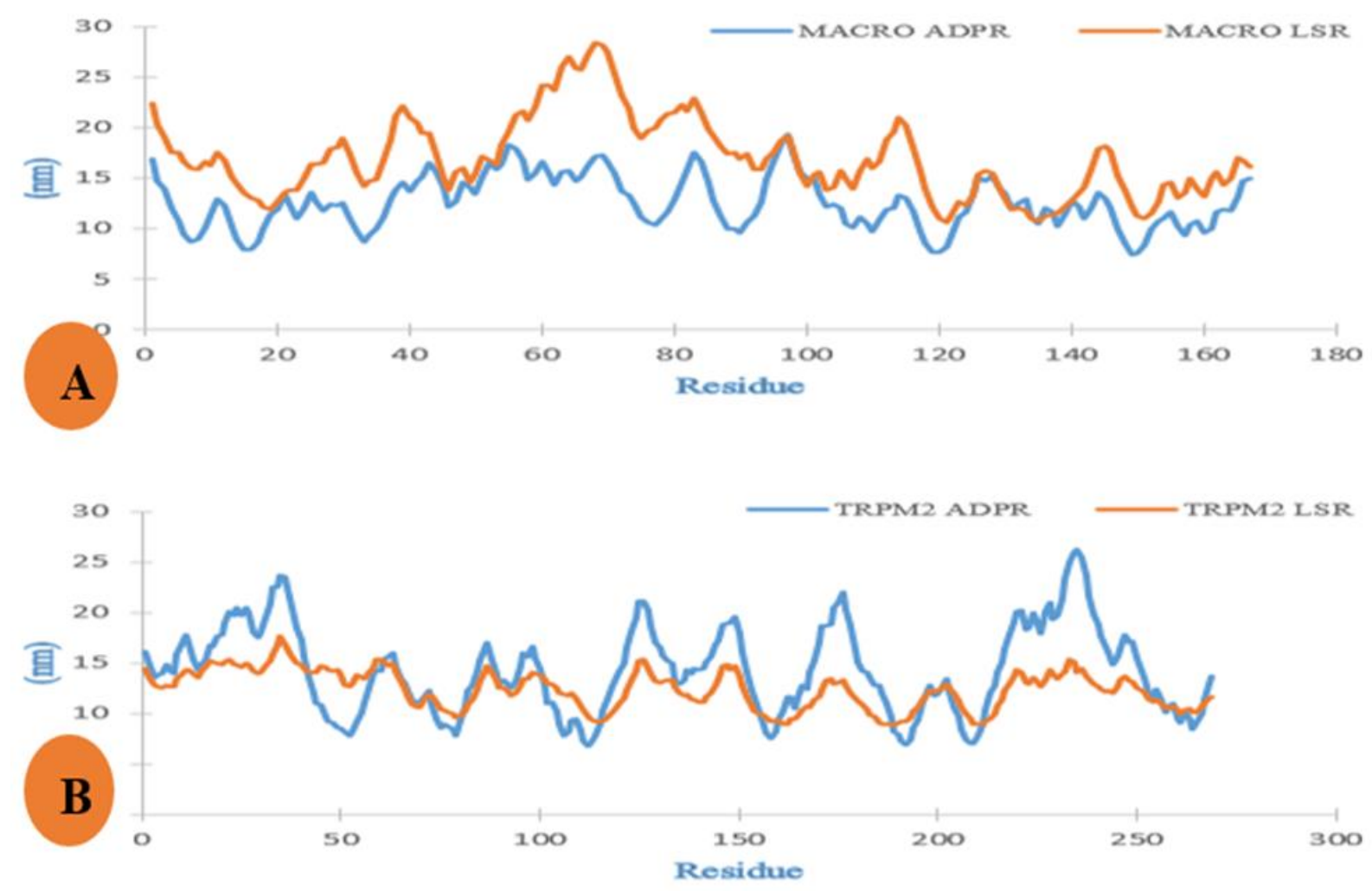

Figure 5. RMSD analyses of viral macrodomain (A) and TRPM2 (B) in complex with ADPR and Losartan. 

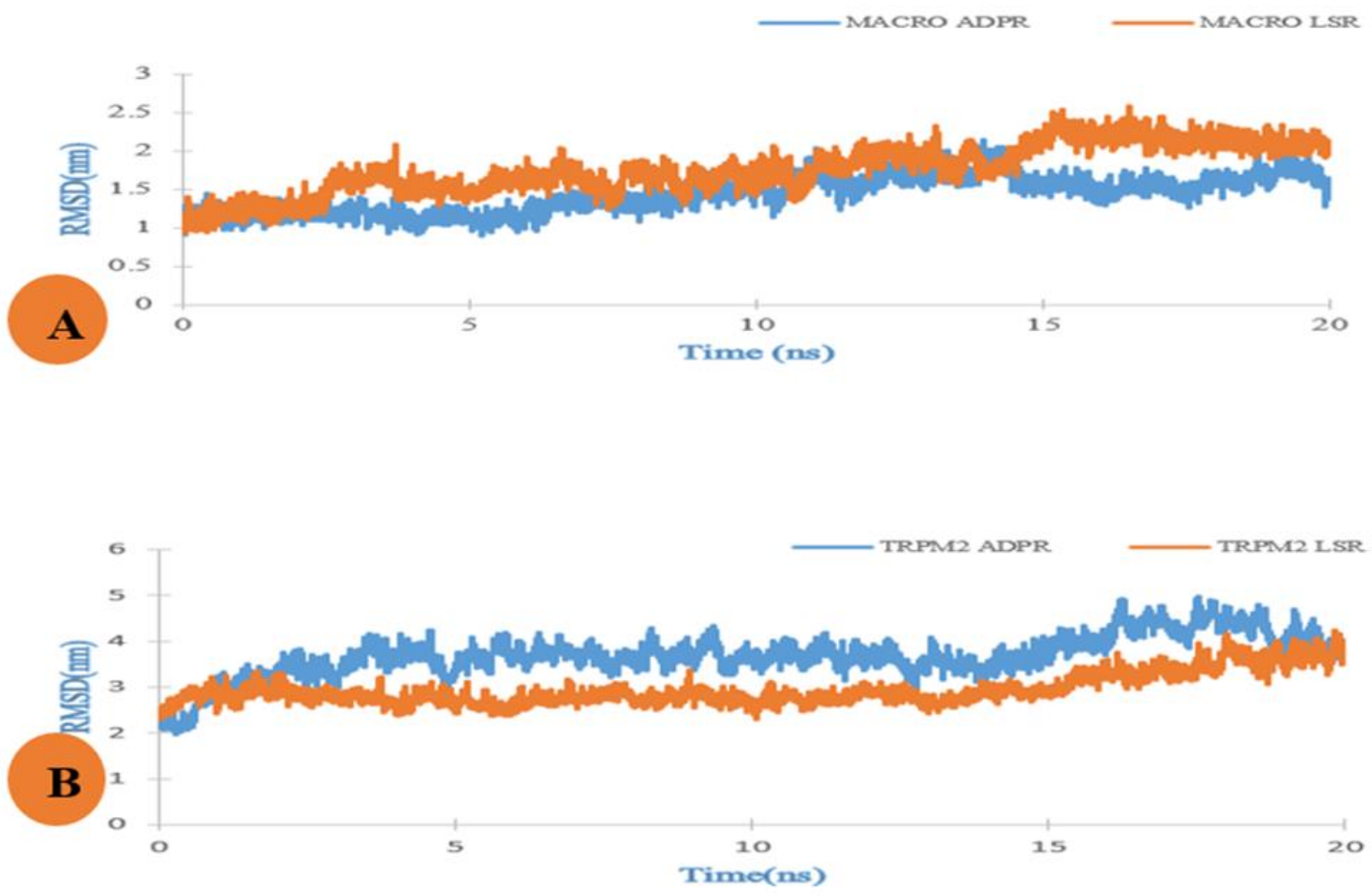

Figure 6. RMSF analyses of macrodomain (A) and TRPM2 (B) in complex with ADPR and LSR ligands. 


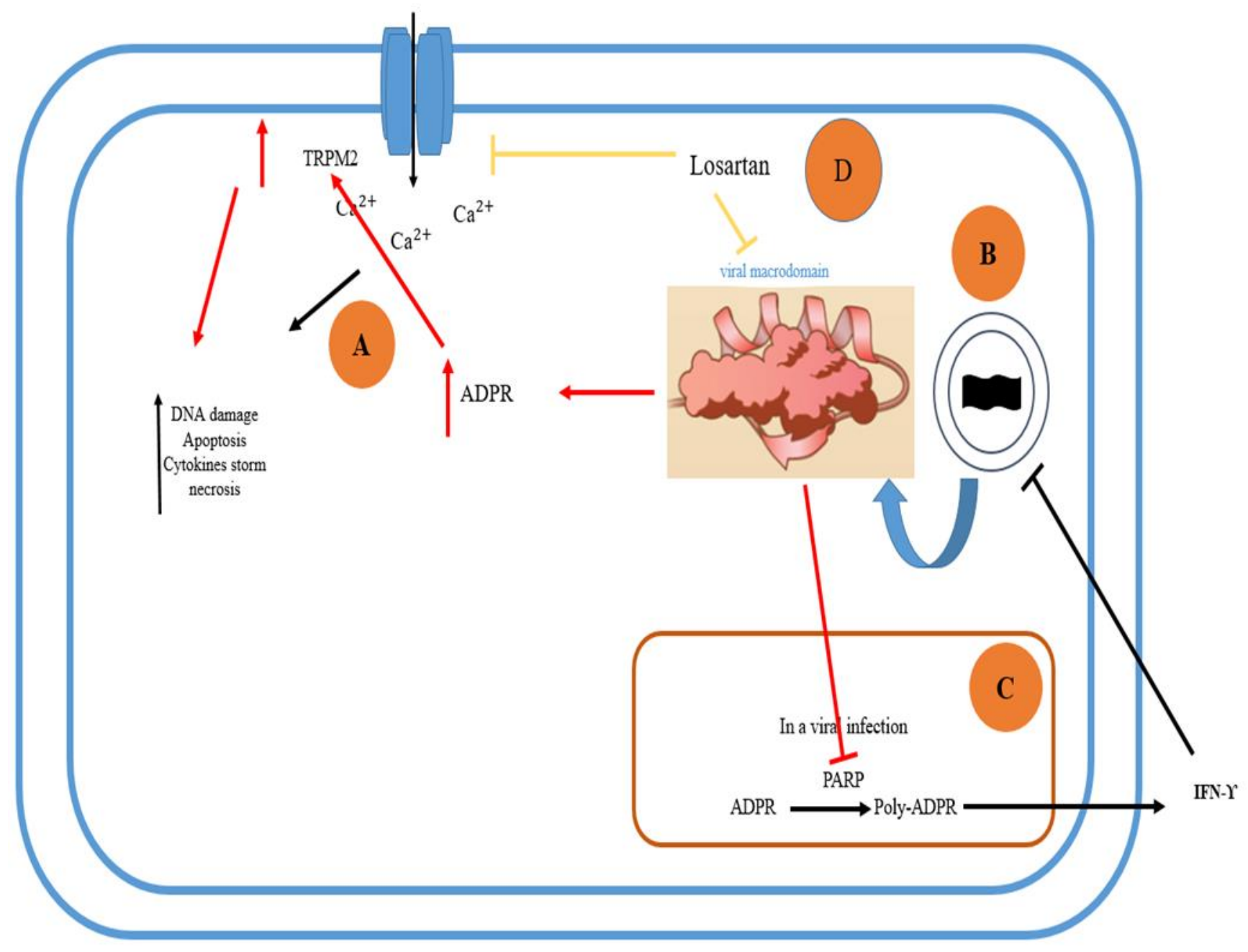

Figure 7. A-Black arrows are the cell mechanism in the normal situation (health people): TRPM2 can active running of the cell death, and apoptosis signaling cascade. This protein is active in the high level of the ADPR in the cell. C-in a viral infection PARP enzyme active in the viral infection. PARP creates poly-ADPR and ADP-ribosylate different types of the protein in the cell. ADP-ribosylating of the proteins is the initiation for the Interferon gamma (IFN $\gamma$ ) signaling cascade, so IFN $\gamma$ produce and release from the cell. The IFN $\gamma$ can active the human cellular immune response against the virus, and increase the resistance of the human cell in confronting with covid-19 virus. B- after the interring to the cell, virus RNA convert to the viral nsps proteins. One of these nsps is the macrodomain which degrade poly-ADPR from the protein, so prevent the IFN $\gamma$ signaling in the cell, and increase ADPR volume in the cell. A-Red arrows show that increasing of ADPR in the cell active the TRPM2 and its signaling cascade consequence of its activating, so cytokine storm, and apoptosis signaling cascade will initiate. D-losartan can bind to the viral macrodomain, and human TRPM2 and prevent their function, therefore cytokine storm will halt, and human cellular immune response will increase 